\title{
Dot trajectories in the superposition of random screens: analysis and synthesis
}

\author{
Isaac Amidror \\ Laboratoire de Systèmes Périphériques, Ecole Polytechnique Fédérale de Lausanne, 1015 Lausanne, Switzerland
}

Received September 4, 2003; revised manuscript received January 15, 2004; accepted February 9, 2004

\begin{abstract}
Moire effects that occur in the superposition of aperiodic layers such as correlated random dot screens are known as Glass patterns. One of the most interesting properties of such moiré effects, which clearly distinguish them from their periodic counterparts, is undoubtedly the appearence in the superposition of intriguing microstructure dot alignments, also known as dot trajectories. These dot trajectories may have different geometric shapes, depending on the transformations undergone by the superposed layers. In the case of simple linear transformations such as layer rotations or layer scalings, the resulting dot trajectories are rather simple (circular, radial, spiral, elliptic, hyperbolic, linear, etc.); but in more complex layer transformations the dot trajectories can have much more interesting and surprising shapes. A full mathematical analysis of the dot trajectories, their morphology, and their various properties is provided. Furthermore, it is shown how the approach also allows us to synthesize correlated random screens that give in their superposition dot trajectories having any desired geometric shapes. Finally, it is also explained why such dot trajectories are visible only in superpositions of aperiodic screens but not in superpositions of periodic screens. (C) 2004 Optical Society of America
\end{abstract}

OCIS codes: 120.4120 .

\section{INTRODUCTION}

It is a well-known fact that the superposition of periodic layers (such as line gratings or dot screens) may give rise to new periodic structures that do not exist in any of the individual layers [see, for example, Fig. 1(d)]. These periodic structures, called moiré effects, have been thoroughly studied in the past, ${ }^{1-3}$ and their mathematical foundations are today fully understood. ${ }^{4}$ The same is also true for moiré effects between repetitive layers (i.e., between geometric transformations of periodic layers).$^{5-10}$ It is also known that the superposition of aperiodic layers, such as random dot screens, may give rise to a different type of moiré pattern, which consists of a single structure resembling a top-viewed funnel or a distant galaxy in the night sky [see, for example, Figs. 1(c) and 1(e)]. This phenomenon is known in literature as a Glass pattern, after Leon Glass, who described it in the late 1960s. ${ }^{11,12}$ These Glass patterns are often surrounded by typical microstructure dot arrangements, known as dot trajectories, that have various shapes such as circles, radial lines, spirals, hyperbolas, and ellipses, depending on the case (see Fig. 1).

Glass patterns in the superposition of random dot screens remained for a long period much less understood than moiré effects that occur between periodic or repetitive layers, partly because they did not easily lend themselves to the same mathematical tools that so nicely explained the classical moiré effects between periodic or repetitive layers (geometric considerations, indicial equations, Fourier series developments, etc.). Only recently has it been shown that in spite of their different appearance, moiré patterns between periodic or repetitive layers and Glass patterns between aperiodic layers are, in fact, particular cases of the same basic phenomenon, and all of them satisfy the same fundamental rules. ${ }^{13-15}$

These recent results basically concern the macroscopic phenomena that occur in the layer superpositions (the Glass or moiré patterns, their behavior under layer transformations, and their intensity profiles as determined by the Fourier theory). Nevertheless, several questions concerning the microstructure dot arrangements (the dot trajectories) that accompany these phenomena in the superposition still remain open. These questions concern, in particular, the mathematical interpretation of the dot trajectories, their morphology, and the reasons that they are visible only in superpositions of aperiodic screens and not in superpositions of periodic screens.

In the present paper we focus our attention on these dot trajectories, and a mathematical explanation is provided regarding their origin, their nature, their morphology, and their various properties. Furthermore, it will be shown how our mathematical understanding of these phenomena allows us not only to analyze the dot trajectories that appear in any given superposition but also to synthesize dot screens that give in their superposition dot trajectories having any desired morphology. Finally, on the basis of our results it will also be explained why such dot trajectories are not visible in superpositions of periodic layers.

It should be noted that in the present study we are not interested in the dot shapes of the individual layers and in their influence on the macroscopic moiré intensity profiles, as described in Ref. 15, and we concentrate only on the dot trajectories that accompany these phenomena. These two approaches are therefore complementary, and they can be used either independently of each other or combined (for example, for synthesizing a Glass pattern 




(a)

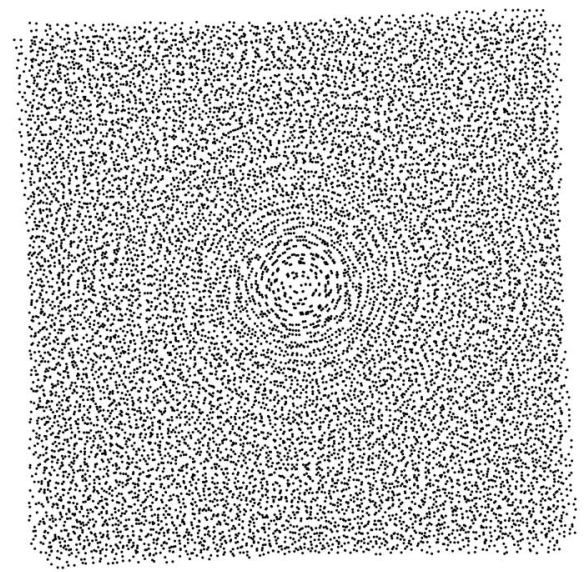

(c)

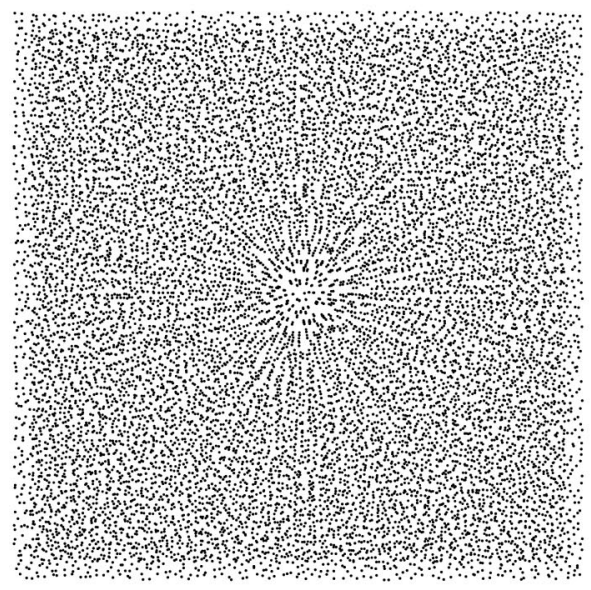

(e)

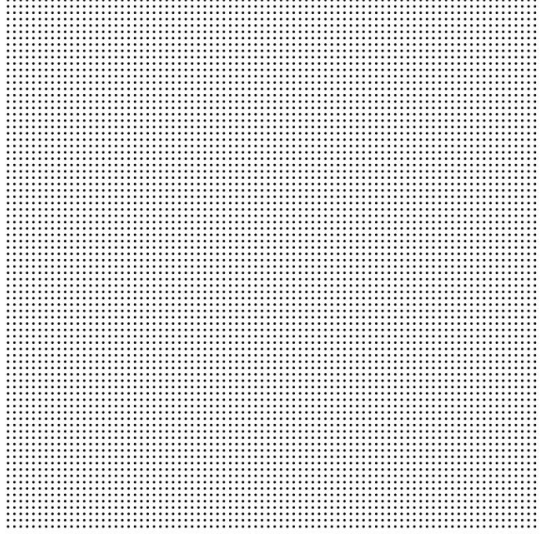

(b)

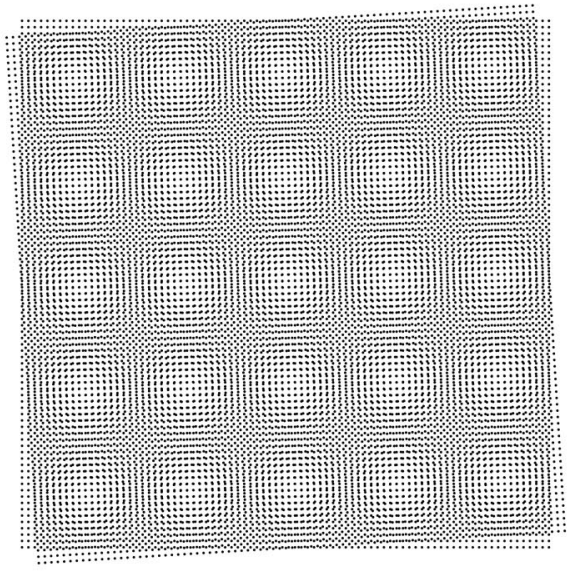

(d)

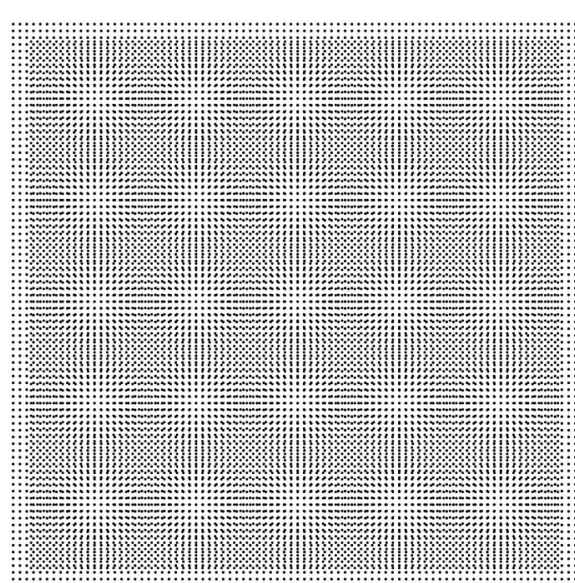

(f)

Fig. 1. (continues on next page). Glass patterns between aperiodic dot screens and their moiré counterparts between periodic dot screens. The aperiodic dot screen (a) and its periodic counterpart (b) were used for generating all the superpositions shown in this figure. (c) The superposition of two identical copies of aperiodic dot screen (a) with a small angle difference gives a Glass pattern about the center of rotation. Note the Glass pattern's typical microstructure consisting of concentric circular dot trajectories. (d) When the superposed layers are periodic, a periodic moiré pattern is generated instead of the Glass pattern. (e) Same as (c), but with a small scaling difference rather than a small angle difference between the superposed layers. In this case the microstructure consists of radial dot trajectories. (f) The periodic counterpart of (e).

having a specified intensity profile, which is surrounded by dot trajectories with a given morphology).
The present paper is structured as follows: Section 2 introduces the terminology and the basic notions that will 


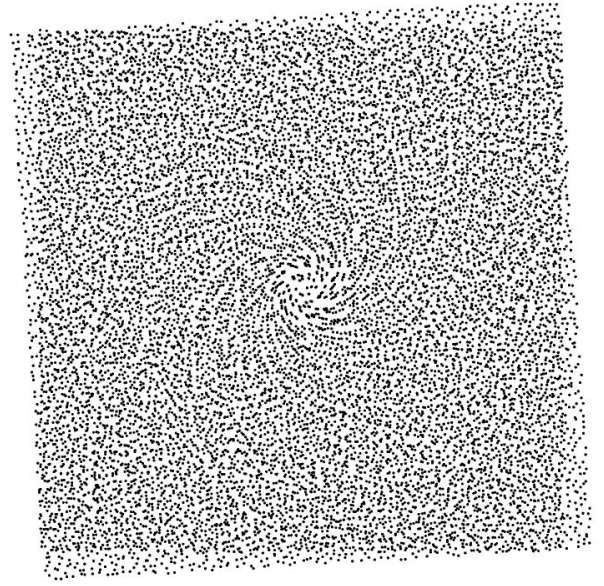

(g)

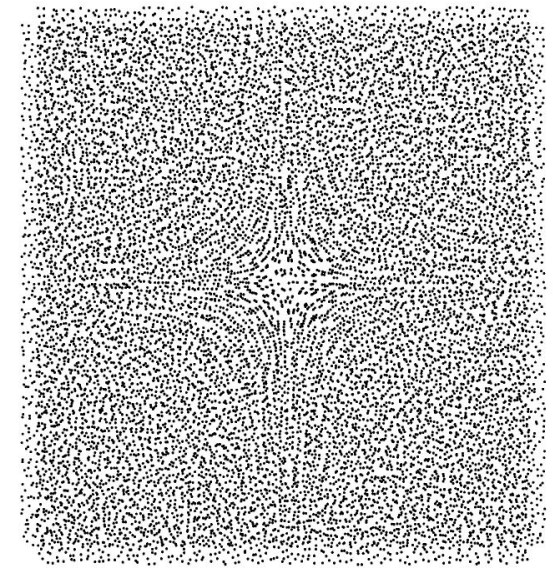

(i)



(k)



(h)

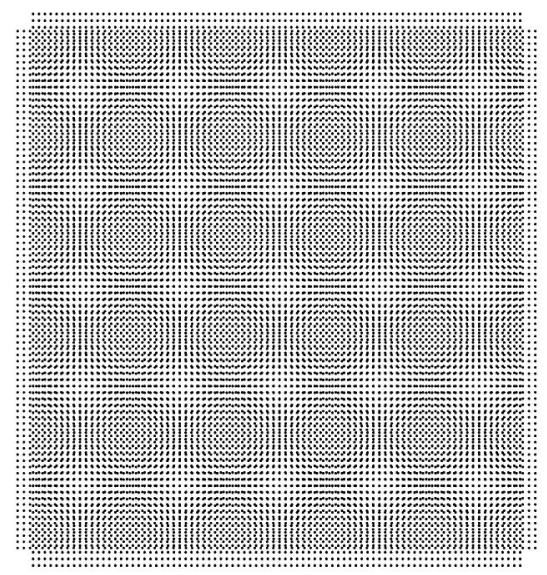

(j)

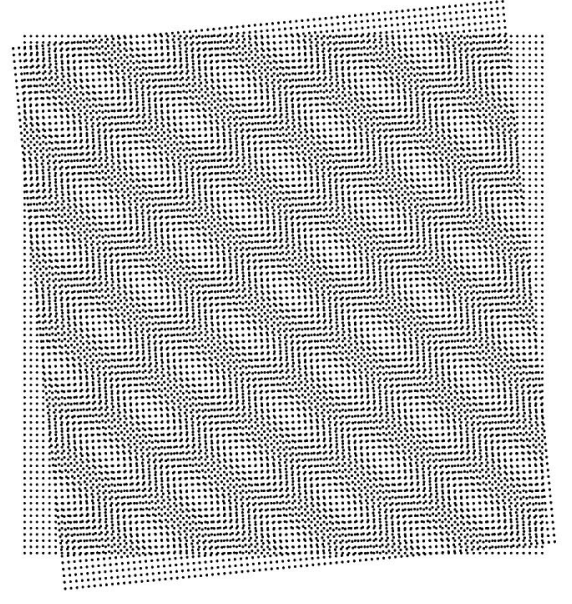

(l)

Fig. 1. (continued). (g) Same as (c) and (e), but with both a small angle and a small scaling difference between the superposed layers. In this case the microstructure consists of spiral dot trajectories. (h) The periodic counterpart of (g). (i) Same as (e), but here the scaled layer undergoes unequal scaling rates in the $x$ and $y$ directions $\left(0<s_{x}<1, s_{y}>1\right)$. Note the hyperbolic dot trajectories in the superposition. (j) The periodic counterpart of (i). (k) Same as (i), but with a small angle difference, too, between the superposed layers. In this case the microstructure consists of elliptic dot trajectories. (l) The periodic counterpart of (k).

be needed for the rest of the paper. Section 3 describes the superposition of aperiodic layers and explains its main properties. Then, Sections 4 and 5 explain the mathematical meaning of the dot trajectories and show how to analyze and how to synthesize them. Finally, Section 6 explains why dot trajectories are observed only in 
aperiodic superpositions and not in their periodic counterparts, and Section 7 presents the main conclusions.

Remark: The PostScript files that generate the dot screens used in the figures of this paper are available on the internet. ${ }^{16}$ They can be downloaded and printed on transparencies by using any standard PostScript printer. Superposing these transparencies manually with varying orientations, shifts, etc., can give a vivid demonstration of the Glass patterns and their dynamic behavior in the superposition, beyond the few static figures that illustrate this paper.

\section{BACKGROUND AND BASIC NOTIONS}

In this introductory section we briefly review the basic notions and terminology that will be used later. Since we will be dealing throughout this paper with layers and layer superpositions, let us start by explaining these notions and their main properties. In fact, a "layer" (or "image") is the most general term we use to cover "anything" in the image domain. It can be periodic or not, continuous or binary, etc. However, we still need to make some basic assumptions regarding our layers.

First of all, we limit ourselves here to monochrome, black-and-white images. This means that each image can be represented by a reflectance function $r(x, y)$, which assigns to any point $(x, y)$ of the image a value between 0 and 1 representing its light reflectance: 0 for black (i.e., no reflected light), 1 for white (i.e., full light reflectance), and intermediate values for in-between shades. In the case of transparencies, the reflectance function is replaced by a transmittance function defined in a similar way.

A superposition of such images can be obtained by overprinting or by laying printed transparencies on top of each other. Since the superposition of black and any other shade always gives black, this suggests a multiplicative model for the superposition of monochrome images. Thus, when two monochrome images are superposed, the reflectance of the resulting image is given by the product of the reflectance functions of the individual images: $r(x, y)=r_{1}(x, y) r_{2}(x, y)$.

Let us now explain what we mean by periodic and by aperiodic or stochastic layers. A one-dimensional function $f(x)$ is said to be periodic if there exists a nonzero number $p$ such that for any $x \in \mathrm{R}, f(x+p)=f(x)$. Similarly, a two-dimensional (2D) layer $r(x, y)$ is said to be periodic if there exists a nonzero vector $\mathbf{p}=\left(p_{1}, p_{2}\right)$ such that for any $(x, y) \in \mathbb{R}^{2}, r\left(x+p_{1}, y+p_{2}\right)$ $=r(x, y)$. If there exist two independent vectors having this property, $r(x, y)$ is said to be two-fold periodic. A layer $r(x, y)$ is said to be aperiodic if it is not periodic. For example, the image of a human portrait or a natural landscape is aperiodic. A random dot screen consisting of randomly positioned black dots is also aperiodic. Note, however, that this random dot screen may also be regarded as a stochastic layer, from a more statistical point of view, if we consider the screen in question as just one possible realization of a stochastic process that has some given statistical distribution. In the context of random dot screens we often use the terms aperiodic layer, stochastic layer, and random layer as synonyms.
Next, let us introduce the notions of macrostructure and microstructure. It is well known that when periodic layers (line gratings, dot screens, etc.) are superposed, new structures of two distinct levels may appear in the superposition that do not exist in any of the original layers: macrostructures and microstructures (Ref. 4, Chap. 8). The macrostructures, also known as moiré patterns, are much coarser than the detail of the original layers, and they are clearly visible even when observed from a distance. The microstructures, on the contrary, are almost as small as the periods of the original layers (typically, just 2-5 times larger), and therefore they are visible only when one is examining the superposition from a close distance or through a magnifying glass. These tiny structures are also called rosettes, owing to the various flowerlike shapes they often form in the superposition of periodic dot screens. Similar phenomena exist also in the superposition of aperiodic or stochastic layers, where both macrostructures and microstructures may become visible. Here, too, macrostructures can be clearly seen when observed from a distance, whereas microstructure dot arrangements (such as dot trajectories; see Section 3 below) are visible only when one is examining the superposition from a close distance.

Note that the distinction between macrostructures and microstructures may seem at first quite artificial and subjective. But in reality, macrostructures and microstructures are simply two different facets of the same phenomenon in the layer superposition. In fact, they only represent two different scales at which we consider the same phenomenon: In the macroscopic scale we consider the global, average behavior of the phenomenon (much like the rules of classical physics), whereas in the microscopic scale we study the behavior of the same phenomenon from the point of view of the individual screen elements and the interactions between them (much like the study of the same physical rules through the behavior of molecules and atoms). ${ }^{17}$

Finally, for the sake of simplicity we consider here only layers having a uniform distribution of their microstructure elements (and hence a constant mean gray level), although our results hold also in more complex structures such as halftone gradations and halftoned images with varying gray levels.

\section{SUPERPOSITION OF APERIODIC LAYERS}

While the superposition of two similar periodic layers generates moiré effects that are themselves periodic, the superposition of two similar aperiodic layers generates an aperiodic moiré effect known as a Glass pattern (compare the right- and the left-hand-side images in each row of Fig. 1). This moiré pattern is typically concentrated around a certain point in the superposition (which is the fixed point of the underlying layer transformation ${ }^{13}$ ), and unlike periodic moirés, it gradually disappears as we go farther away from this point. Depending on whether it was obtained by rotation of one of the superposed layers, by a scaling transformation, or by a combination of the two, it gives rise to an intriguing ordering of the microstructure elements in the superposition in dot trajectories 
having a circular, radial, or spiral shape [see Figs. 1(c), $1(\mathrm{e})$, and $1(\mathrm{~g})$ ] $^{12}$ Other layer transformations may give rise to Glass patterns having elliptic, hyperbolic, or other geometrically shaped trajectories [Figs. 1(i) and $1(\mathrm{k}){ }^{12}$ However, when we rotate one of the aperiodic layers by $180^{\circ}$ [see Figs. 2(a) and 2(b)], the Glass pattern disappears.

As already explained by Glass, this phenomenon occurs thanks to the local correlation between the structures of the two superposed layers; in fact, its intensity can be used as a visual indication of the degree of correlation between the two layers at each point of the superposition. Thus, when two identical layers having the same arbitrary structure are slightly rotated on top of each other [see Fig. 1(c)], a visible Glass pattern is generated around the center of rotation (the fixed point), indicating the high correlation between the two layers in this area. Within the center of this Glass pattern the corresponding elements from the two layers fall almost exactly on top of each other, but slightly away from the center they fall just next to each other, generating circular trajectories of point pairs. Farther away from the center the correlation between the two layers becomes smaller and smaller, and the elements from both layers fall in an arbitrary, noncorrelated manner; in this area the Glass pattern is no longer visible. This explains why the Glass pattern gradually decays and disappears as we go away from its center. Note, however, that when the two superposed layers are not at all correlated, no Glass pattern appears in the superposition [this is, indeed, what happens when we rotate one of the aperiodic transparencies by $180^{\circ}$ on top of its identical copy, as shown in Fig. 2(a)]. In intermediate cases, where the two superposed layers are only partially correlated, the Glass pattern becomes weaker and less perceptible, depending on the degree of the correlation that still remains between the superposed layers.

As we can see, the explanation above is based on an observation of the individual elements of the original layers and their behavior in the superposition. We say, therefore, that this explanation is based on the microstructure. To obtain the point of view of the macrostructure, we have to look at the layers and their superposition from a greater distance, where the individual elements of the layers are no longer discerned by the eye and what we see is only an integrated gray-level average of the microstructure in each area of the superposition. From the point of view of the macrostructure, the center of the Glass pattern consists of a brighter gray level than areas farther away (owing to the partial overlapping of the microstructure elements of the two layers in this area); farther away, the macroscopic gray level is darker (because elements from the two layers are more likely to fall side by side, thus increasing the covering rate and the macroscopic gray level). This means that the Glass pattern is not just an optical illusion, and it corresponds, indeed, to the physical reality. In fact, just like in the periodic case (see Proposition 8.1 in Ref. 4), moiré patterns are simply the macroscopic interpretation of the variations in the microstructures throughout the superposition.

On the other hand, the ordering of the microstructure elements within a Glass pattern into circular, radial, or spiral dot trajectories is no longer visible from far away (try to observe the images in the left column of Fig. 1 from a distance of 3-4 m, where the individual elements of the layers are no longer discerned by the eye). These dot trajectories are not part of the macrostructure description, and they belong to the microstructure of the superposition, just like rosettes in the periodic case. And indeed, from the point of view of the macrostructure there is no distinction between gray levels obtained when the neighboring elements in the superposition are located on circular trajectories, owing to rotation, or on radial trajectories, owing to a scaling transformation: What counts in both cases is the resulting mean coverage rate, which determines the overall gray level, and not the specific geometric arrangement of the dots.

In the present paper we focus our attention on the microstructure of the Glass patterns and on the arrangement of the individual dots into dot trajectories. It is important to note that Fourier analysis methods are not applicable here, since they treat only the global aspects of the superposition but do not go down to the level of the

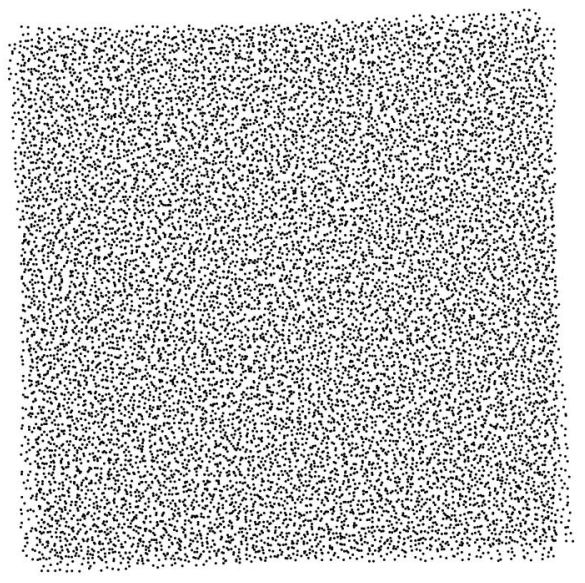

(a)



(b)

Fig. 2. (a) and (b) Same as (c) and (e) in Fig. 1, but with one of the layers being rotated by $180^{\circ}$; in this case the Glass patterns disappear completely. 
individual screen dots and their local behavior. ${ }^{18}$ In the following section we will see what mathematical tools can be used to investigate these microscopic phenomena.

\section{MORPHOLOGY OF THE MICROSTRUCTURES: ANALYSIS OF DOT TRAJECTORIES}

One of the most interesting aspects of aperiodic moiré effects, first mentioned by Glass, ${ }^{12}$ concerns the morphology of the microstructures that are generated in the superposition of aperiodic screens. As we can see in many of the figures throughout the present paper, fixed points in the superposition of aperiodic screens are often surrounded by dot trajectories having various geometric shapes, such as circles, ellipses, hyperbolas, or spirals, etc., depending on the case. For example, a fixed point that occurs as a result of a rotation of one of the superposed layers is typically surrounded by circular dot trajectories [Fig. 1(c)], while a fixed point due to a uniform scaling transformation is typically surrounded by radial dot trajectories [Fig. 1(e)]. Spiral dot trajectories, on their part, are typical of intermediate cases involving both rotation and scaling [Fig. 1(g)], and hyperbolic dot trajectories typically occur around the fixed point in scaling transformations involving expansion along one direction and contraction along a different direction [Fig. 1(i)]. Some interesting dot trajectories that are generated by nonlinear transformations of the original aperiodic layers are shown in Fig. 3. Note that dot trajectories may appear even in superpositions where no fixed points exist [see, for example, Fig. 3(d)]. This large versatility in the dot trajectories and in their shapes certainly deserves investigation. It is therefore our aim here to study the dot trajectories, their morphology, and their various properties.

Suppose, to begin with, that we superpose two identical aperiodic screens exactly on top of each other and that we apply to one of the superposed screens a transformation $\mathbf{g}(x, y)$ given by

$$
x^{\prime}=g_{1}(x, y), \quad y^{\prime}=g_{2}(x, y) .
$$

(Note that $x^{\prime}$ and $y^{\prime}$ do not represent derivatives but rather a different coordinate system in the plane.) As a simple example, we may think of the linear transformation:

$$
x^{\prime}=a_{1} x+b_{1} y, \quad y^{\prime}=a_{2} x+b_{2} y .
$$

The dot trajectories that are generated in these superpositions are physically made of pairs of dots, one dot from each layer. Each such pair in the superposition represents, in fact, two successive locations of the same dot, namely, the dot's location before and after the layer transformation $\mathbf{g}(x, y)$ has been applied. Let us try to see how these dot trajectories can be analyzed mathematically.

\section{A. Dot Trajectories As Solution Curves of a System of Differential Equations}

A first attempt to interpret such dot trajectories has already been presented in Ref. 12 . According to this approach, we consider the finite transformation $\mathbf{g}(x, y)$ as an iteration of an infinitesimal transformation. This reasoning leads one from Eqs. (1) to a pair of first-order dif- ferential equations that correspond to our infinitesimal transformation:

$$
\frac{\mathrm{d}}{\mathrm{d} t} x(t)=g_{1}(x(t), y(t)), \quad \frac{\mathrm{d}}{\mathrm{d} t} y(t)=g_{2}(x(t), y(t)) .
$$

For example, in the particular case of linear transformation (2), we get

$$
\begin{aligned}
& \frac{\mathrm{d}}{\mathrm{d} t} x(t)=a_{1} x(t)+b_{1} y(t), \\
& \frac{\mathrm{d}}{\mathrm{d} t} y(t)=a_{2} x(t)+b_{2} y(t) .
\end{aligned}
$$

The solution of system (3) consists of a family of curves in the $x, y$ plane, whose members differ from each other by some constants $c$ (Ref. 19, pp. 180-186). The parametric representation of each of these curves is $[x(t), y(t)]$, where the parameter $t$ may be thought of as time. Each of these solution curves is called a trajectory since it traces out the evolution of the curve as $t$ is being varied.

According to this reasoning, ${ }^{12}$ the dot trajectories observed in the superposition of aperiodic dot screens simply represent the trajectories (i.e., the solution curves) of this underlying pair of differential equations. ${ }^{20}$ And, indeed, this explanation agrees with experimental evidence in various cases.

However, a deeper examination of the question shows that this reasoning is not always true. This is clearly demonstrated by the following examples.

Example 1. Consider the identity transformation $\mathbf{g}(x, y)=(x, y)$, which is a particular case of Eqs. (2) with $a_{1}=b_{2}=1$ and $a_{2}=b_{1}=0$. Obviously, in this case the two layers perfectly coincide on top of each other, and no dot trajectories are generated. However, the solution of the corresponding system of differential equations (4) with $a_{1}=b_{2}=1$ and $a_{2}=b_{1}=0$ gives the family of straight lines $y=c x$ for any constant $c$, and its solution curves (trajectories) consist of radial lines emanating from the origin (see Ref. 19, p. 168). While such radial dot trajectories can be expected in the layer superposition when the mapping being applied to one of the screens is a scaling by $s \neq 1$ [see Fig. 1(e)], it is clear that in our present case, where $s=1$, no dot trajectories will appear in the superposition.

Example 2. Suppose that we apply to one of the two superposed layers a rotation by a small angle $\alpha$, as illustrated in Fig. 1(c). This linear transformation is clearly a particular case of Eqs. (2), with $a_{1}=\cos \alpha, b_{1}=-\sin \alpha$, $a_{2}=\sin \alpha$, and $b_{2}=\cos \alpha$. According to Fig. 1(c) we would expect the solution curves of differential equations (4) to consist of circular trajectories about the center of rotation. However, a short verification shows that this is not always the case. Although for $\alpha=90^{\circ}$, where $a_{1}$ $=b_{2}=0, a_{2}=1$ and $b_{1}=-1$, the differential equations do have circular trajectories as expected, it turns out that for $\alpha=0^{\circ}$ (the identity transformation, where $a_{1}$ $=b_{2}=1$ and $a_{2}=b_{1}=0$ ) the solution curves of the differential equations consist of a family of radial lines emanating from the origin. Furthermore, for all intermediate rotation angles $0^{\circ}<\alpha$ $<90^{\circ}$ the solution curves consist of a family of spirals 




(a)

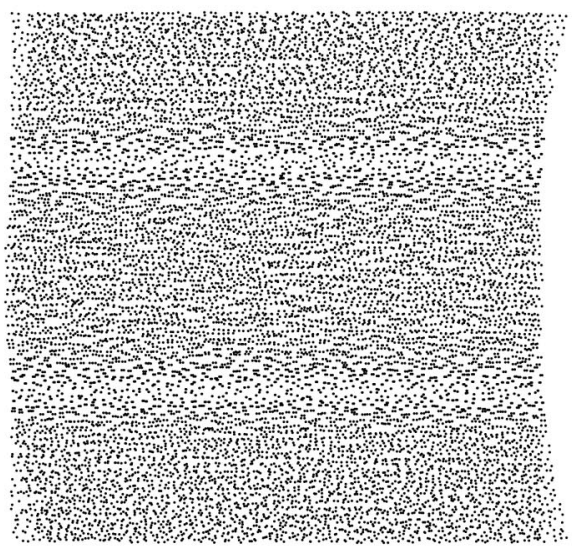

(c)

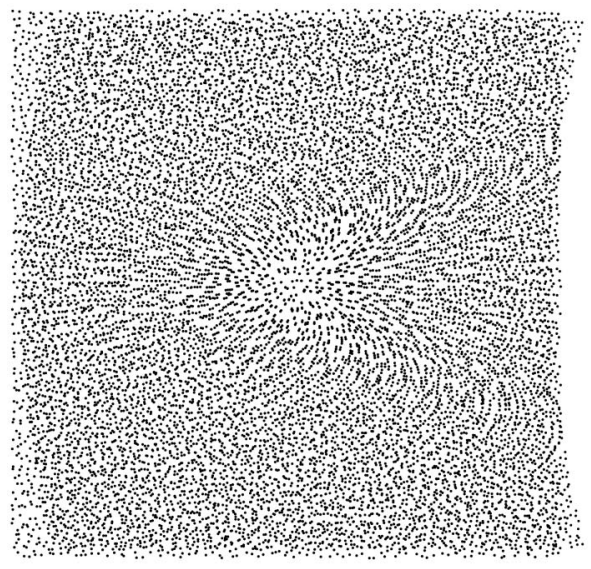

(e)

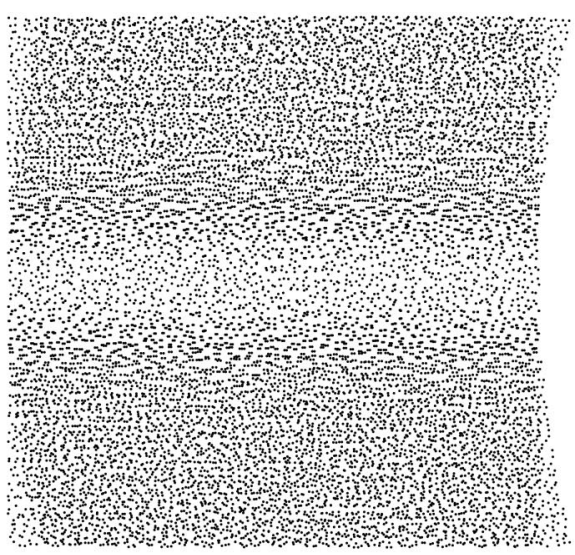

(b)

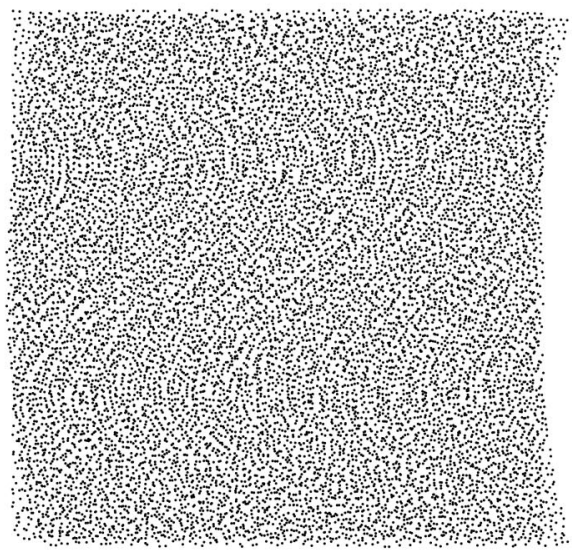

(d)

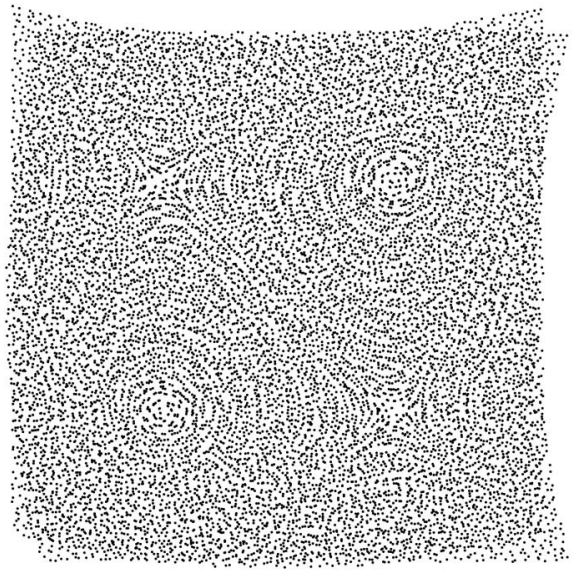

(f)

Fig. 3. (a) Aperiodic dot screen that has undergone the parabolic transformation $\mathbf{g}(x, y)=\left(x+a y^{2}, y\right)$. (b) The superposition of two identical aperiodic dot screens, one of which has undergone the parabolic transformation $\mathbf{g}(x, y)$. Since this transformation does not involve layer shifts, the two layers clearly coincide along the $x$ axis. (c) The superposition of two identical aperiodic dot screens, one of which has undergone the parabolic transformation $\mathbf{g}(x, y)=\left(x+a y^{2}, y\right)$, while the untransformed screen has been slightly shifted by $x_{0}$. A pair of linear Glass patterns is generated in their superposition. (d) Same as in (c) but with a slight layer shift of $\left(x_{0}, y_{0}\right)$ rather than $x_{0}$. (e) The superposition of two identical aperiodic dot screens, one of which has undergone the parabolic transformation $\mathbf{g}(x, y)=\left(x+a y^{2}, y\right)$, while the untransformed layer has been slightly scaled up. (f) An example with four fixed points: the superposition of two identical aperiodic dot screens, one of which has undergone a vertical parabolic transformation plus a slight vertical shift of $y_{0}$ downward, while the other has undergone a horizontal parabolic transformation plus a slight horizontal shift of $x_{0}$ to the left. Note the two circular and the two hyperbolic Glass patterns that are clearly visible about the fixed points in the superposition. 
(see, for example, Ref. 21, p. 144); as $\alpha \rightarrow 90^{\circ}$ these spirals gradually approach circles, but as $\alpha \rightarrow 0^{\circ}$, i.e., for small rotation angles, the spirals straighten out and gradually approach radial straight lines. This result does not agree with our expectations based on Fig. 1(c).

These examples clearly show that the traditional approach based on the system of differential equations (4) does not correctly correspond to the dot trajectories that appear in our layer superposition when the mapping (2) is applied to one of the superposed layers. The reasons for this failure as well as ways to overcome it will become clear shortly.

\section{B. Dot Trajectories As a Vector Field}

A better understanding of this problem can be obtained by considering the situation from another point of view. As explained in Appendix A, any two-dimensional transformation $\mathbf{g}(x, y)$ can be also interpreted as a vector field that assigns to each point $(x, y)$ in the $x, y$ plane the vector $\mathbf{g}(x, y)$. This vector field can be illustrated graphically by drawing starting from each point $(x, y)$ an arrow having the length and the orientation of the vector $\mathbf{g}(x, y)$. This interpretation has the important advantage of clearly showing in a visual way the effect of the transformation $\mathbf{g}(x, y)$ on any point of the $x, y$ plane. It should be noted that the system of differential equations (3) is simply a different representation of the same vector field $\mathbf{g}(x, y)$, since its solution curves $[x(t), y(t)]$ express the field lines (trajectories) of the vector field $\mathbf{g}(x, y)$ (see Appendix A).

Now, remember that the dot trajectories in our superposition of two aperiodic dot screens consist of pairs of dots, which represent the location of a screen dot before and after the layer transformation $\mathbf{g}(x, y)$ has been applied. These dot pairs can be represented, therefore, as a vector field, which assigns to each point $(x, y) \in \mathbb{R}^{2}$ a vector that connects $(x, y)$ to its new location $\mathbf{g}(x, y) \in \mathrm{R}^{2}$ under the transformation $\mathbf{g} .{ }^{22}$ It is important to note, however, that the vector field of the transformation $\mathbf{g}(x, y)$ itself does not have this property; that is, the vector it assigns to $(x, y)$ does not connect $(x, y)$ to its destination $\mathbf{g}(x, y)$ but rather to the point $(x, y)+\mathbf{g}(x, y)$. For example, if we consider the identity transformation $\mathbf{g}(x, y)=(x, y)$ (see example 1 ), it is clear that in this case the vector attached to each point $(x, y)$ is the vector $(x, y)$ itself, which points, therefore, to the point $(2 x, 2 y)$ and not to the destination point under $\mathbf{g}$, which is the point $(x, y)$.

Therefore, in order to obtain a vector field that correctly represents our dot trajectories, we must consider, instead of the transformation $\mathbf{g}(x, y)$ itself (the transformation that has been applied to one of the superposed layers), the relative transformation between the two layers, which is given by

$$
\mathbf{h}(x, y)=\mathbf{g}(x, y)-(x, y) .
$$

And, indeed, if we draw the vector field representation of this transformation, we obtain exactly what we desired: The vector field of $\mathbf{h}(x, y)$ assigns to each point $(x, y)$ the vector $\mathbf{g}(x, y)-(x, y)$, which connects the original point $(x, y)$ to its destination under the layer transformation $\mathbf{g}$, the point $\mathbf{g}(x, y)$.
If we take one step further and allow both of the superposed layers to be transformed, one by a mapping $\mathbf{g}_{1}(x, y)$ and the other by a mapping $\mathbf{g}_{2}(x, y)$, then the relative transformation between the two layers becomes

$$
\mathbf{h}(x, y)=\mathbf{g}_{1}(x, y)-\mathbf{g}_{2}(x, y) .
$$

Note, however, that in this case the dot pairs that make up the dot trajectories in the superposition no longer represent a dot's location before and after the layer transformation has been applied but rather the new locations of the same original dot under the transformation $\mathbf{g}_{1}(x, y)$ and under the transformation $\mathbf{g}_{2}(x, y)$.

It should be also mentioned that since the two dots that compose each dot pair in the layer superposition are identical, the dot pairs (and hence the dot trajectories) remain unchanged when we interchange the transformations undergone by the two layers. In other words, the dot trajectories in the superposition do not show the direction (the positive or negative sense) of the difference vectors.

These results can be therefore summarized as follows:

Proposition 1. Suppose that we are given two identical aperiodic dot screens that are superposed on top of each other in full coincidence, dot on dot. When we apply to one of the layers a transformation $\mathbf{g}(x, y)$, we obtain in the superposition dot trajectories that correspond to the vector field $\mathbf{h}(x, y)=\mathbf{g}(x, y)-(x, y)$ [or, equivalently, to the vector field $\mathbf{h}(x, y)=(x, y)-\mathbf{g}(x, y)]$. Similarly, if we apply $\mathbf{g}_{1}(x, y)$ to one of the layers and $\mathbf{g}_{2}(x, y)$ to the other layer, where both $\mathbf{g}_{1}(x, y)$ and $\mathbf{g}_{2}(x, y)$ are weak transformations, we obtain in the superposition dot trajectories that correspond to the vector field $\mathbf{h}(x, y)$ $=\mathbf{g}_{1}(x, y)-\mathbf{g}_{2}(x, y)$ [or, equivalently, to the vector field $\left.\mathbf{h}(x, y)=\mathbf{g}_{2}(x, y)-\mathbf{g}_{1}(x, y)\right]$.

It should be understood, however, that although such dot trajectories are theoretically generated in the superposition in all cases, they can be clearly visible [and correspond to the vector field $\mathbf{h}(x, y)]$ only if the layer transformations $\mathbf{g}(x, y), \mathbf{g}_{1}(x, y)$, and $\mathbf{g}_{2}(x, y)$ are not too "violent." Otherwise, the correlation between the superposed layers is strongly reduced, and the visual effect in the layer superposition may be lost and no longer correspond to the vector field.

Let us now see a few examples to illustrate how this approach explains the dot trajectories obtained in our aperiodic screen superpositions. We start with the two cases that caused us trouble in examples 1 and 2 .

Example 3. Suppose that one of the superposed screens, say, the first one, is scaled by $s>0$. In order not to lose the correlation between the two screens, we assume that $s \approx 1$. The relative transformation between the two layers is given in this case by

$$
\mathbf{h}(x, y)=(s x, s y)-(x, y)=(s-1)(x, y) .
$$

Regarding this linear transformation as a vector field, we see that it assigns to any point $(x, y)$ in the $x, y$ plane a vector $(s-1)(x, y)$. Clearly, if $s>1$ this vector field consists of radial trajectories emanating from the origin [see Fig. 4(b)], whereas if $0<s<1$ it consists of radial trajectories pointing to the origin [Fig. 4(d)]. The origin itself is a fixed point of this vector field. And, indeed, this fully agrees with the radial dot trajectories that we observe in the screen superposition in both of these cases. 




(a)

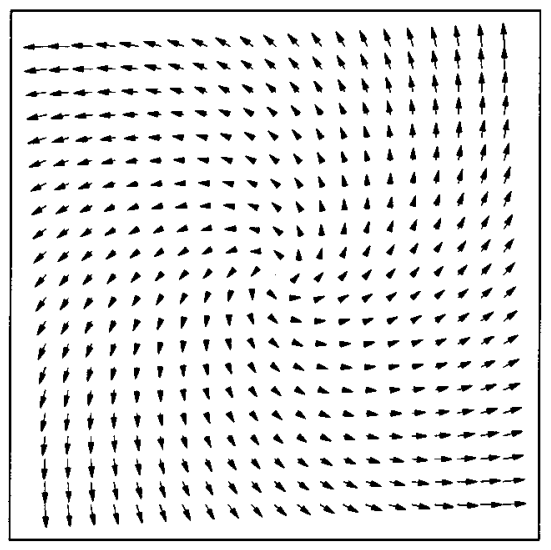

(c)

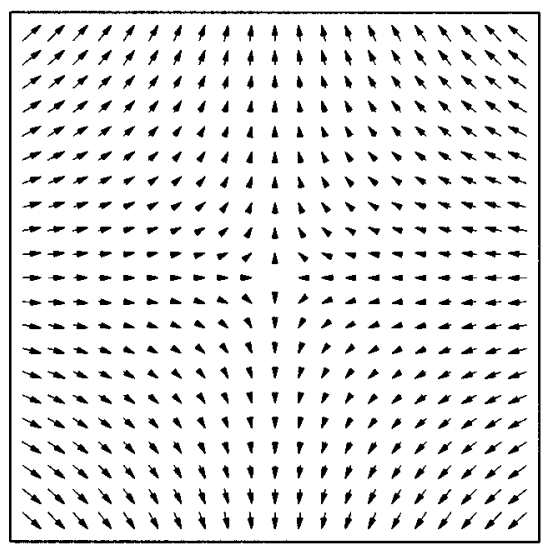

(e)

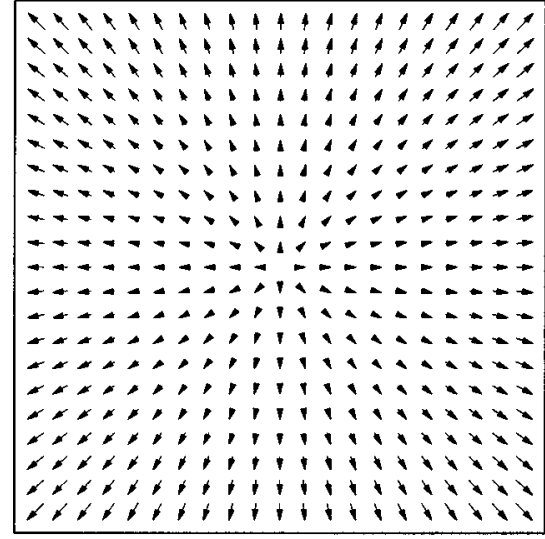

(b)



(d)

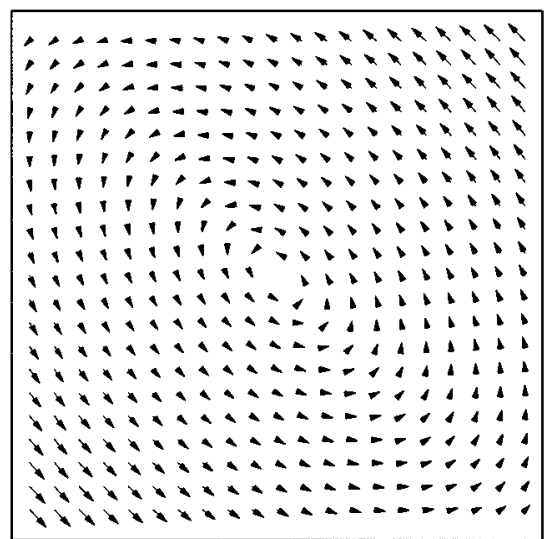

(f)

Fig. 4. Vector field representation of the relative transformation $\mathbf{h}(x, y)=\mathbf{g}(x, y)-(x, y)$ where $\mathbf{g}(x, y)$ (the transformation undergone by the first layer) is (a) a slight rotation; (b) a slight scaling ( $s x, s y$ ) with $s>1$; (c) both a slight rotation and a slight scaling with $s>1$; (d) a slight scaling with $0<s<1$; (e) a slight scaling with $0<s_{x}<1, s_{y}>1$; (f) both a slight scaling with $0<s_{x}<1, s_{y}>1$ and a small rotation. Compare with Figs. 1(c), 1(e), 1(g), 1(i), and 1(k).

Note, however, that the dot trajectories in the screen superposition [Fig. 1(e)] do not show the direction along the trajectories [which is indicated by the arrowheads of Figs. 4(b) and 4(d)].
Finally, let us examine the case of $s=1$, which caused us trouble in example 1 . In this case (scaling by 1 ) our vector field $\mathbf{h}(x, y)$ assigns to each point $(x, y)$ in the $x, y$ plane the zero vector; hence this transformation has no 
fixed points and no trajectories-exactly as we would expect when the two identical screens fully coincide on top of each other. Thus by considering the vector field of the relative layer transformation $\mathbf{h}(x, y)$ we have overcome our troubles from example 1.

Example 4. Let us now consider the linear mapping that corresponds to a rotation of the first layer about the origin by a small angle $\alpha$. In this case the vector field $\mathbf{h}(x, y)=\mathbf{g}(x, y)-(x, y)$ assigns to each point $(x, y)$ in the $x, y$ plane a vector that connects it to its new location after the rotation. This vector field consists of a circular arrangement of arrows (dot pairs) about the origin, as we can see in Fig. 4(a). This agrees, indeed, with the dot trajectories that are generated in the screen superposition, as shown in Fig. 1(c).

These examples show clearly that by considering the vector field of the relative layer transformation $\mathbf{h}(x, y)$, we have overcome our troubles from examples 1 and 2 . Now, remember that the system of differential equations (3) is simply a different representation of the vector field $\mathbf{g}(x, y)$, since its solution curves $[x(t), y(t)]$ express the field lines (trajectories) of the vector field $\mathbf{g}(x, y)$. It can be expected, therefore, that by using the relative layer transformation $\mathbf{h}(x, y)$ instead of $\mathbf{g}(x, y)$ in Eqs. (3) we can also "cure" the differential-equation approach of Subsection 4.A and make it work properly. A short investigation of this point shows indeed that in general the trajectories of the modified differential equations,

$$
\frac{\mathrm{d}}{\mathrm{d} t} x(t)=h_{1}(x(t), y(t)), \quad \frac{\mathrm{d}}{\mathrm{d} t} y(t)=h_{2}(x(t), y(t)),
$$

correspond well to our dot trajectories in the superposition; and yet, in some cases there still exist some minor discrepancies between them. It turns out that the arrow representation of the vector field $\mathbf{h}(x, y)$ fully corresponds to our dot trajectories, because each of the arrows connects the location of the same screen dot in the first and in the second transformed layers. But the solution curves (trajectories) of the modified system of differential equations do not correspond to the motion of a screen dot under the given layer transformation: Although the solution curves of this system of differential equations express mathematically the field lines of the relative layer transformation $\mathbf{h}(x, y)$, these field lines are not always precisely what we are looking for but only a close approximation. This subject is discussed further in Appendix B. It should be mentioned, however, that since we in any case must restrict ourselves to weak layer transformations (in order not to destroy the correlation in the superposition), this discrepancy is marginal and it can be neglected for all practical needs (as we will see, for instance, in example 6 below).

To further illustrate our mathematical interpretation of the dot trajectories in the superposition as a vector field, Figs. 4 and 5 provide a graphical representation of the vector field $\mathbf{h}(x, y)$ for some of the most interesting superpositions shown in Figs. 1 and 3. These cases include both linear and nonlinear layer transformations, and they illustrate dot trajectories that occur around one or more fixed points, as well as dot trajectories that are generated when no fixed points exist in the superposition. Note the perfect agreement between the dot trajectories in each of the superpositions and the arrows showing the dot displacements in the corresponding vector field $\mathbf{h}(x, y)$.

These examples clearly show how the vector field approach presented above explains the dot trajectories that are generated in the superposition of aperiodic screens. It should be remembered, however, that the dot trajectories are visible in the layer superposition owing to the correlation between the superposed layers. Hence if the original layers are not sufficiently correlated, or if the transformations $\mathbf{g}(x, y)$ are too "violent" and destroy the correlation between the layers, no dot trajectories will be seen in the superposition (even if the corresponding vector field still includes visible solution curves).

\section{SYNTHESIS OF DOT TRAJECTORIES}

Having succeeded in analyzing the dot trajectories that occur in the superposition of aperiodic screens, we may ask now whether we can also synthesize such effects. In other words, can we synthesize, for any given vector field $\mathbf{h}(x, y)$, two aperiodic dot screens that generate in their superposition dot trajectories corresponding to $\mathbf{h}(x, y)$ ?

Suppose that we are given a vector field $\mathbf{h}(x, y)$ or, equivalently, a system of differential equations

$$
\frac{\mathrm{d}}{\mathrm{d} t} x(t)=h_{1}(x(t), y(t)), \quad \frac{\mathrm{d}}{\mathrm{d} t} y(t)=h_{2}(x(t), y(t)) .
$$

We wish to synthesize an aperiodic screen superposition whose dot trajectories visually illustrate our given vector field (or system of differential equations).

We start by superposing two identical aperiodic dot screens on top of each other in full coincidence. As we already know from the first part of proposition 1 , if we apply a transformation $\mathbf{g}(x, y)$ to one of the layers we obtain in the superposition the dot trajectories that correspond to $\mathbf{h}(x, y)=\mathbf{g}(x, y)-(x, y)$ [or equivalently to $\mathbf{h}(x, y)$ $=(x, y)-\mathbf{g}(x, y)]$. Therefore, in order to obtain in the superposition dot trajectories which correspond to $\mathbf{h}(x, y)$, we may apply to one of the superposed layers the transformation $\mathbf{g}(x, y)=(x, y)+\mathbf{h}(x, y)$ [or equivalently, $\mathbf{g}(x, y)=(x, y)-\mathbf{h}(x, y)]$, while the other layer remains unchanged.

Similarly, it follows from the second part of proposition 1 that by applying a transformation $\mathbf{g}_{1}(x, y)$ to one of the superposed layers and a transformation $\mathbf{g}_{2}(x, y)$ to the other layer, where both $\mathbf{g}_{1}(x, y)$ and $\mathbf{g}_{2}(x, y)$ are weak transformations, we obtain in the superposition the dot trajectories of $\mathbf{h}(x, y)=\mathbf{g}_{1}(x, y)-\mathbf{g}_{2}(x, y)$. This gives us an alternative way to synthesize dot trajectories that correspond to $\mathbf{h}(x, y)$, by applying to both of the superposed layers transformations $\mathbf{g}_{1}(x, y)$ and $\mathbf{g}_{2}(x, y)$ whose difference gives $\mathbf{h}(x, y)$. For example, we may distribute the deformation in equal parts between the two layers as follows:

$$
\begin{aligned}
& \mathbf{g}_{1}(x, y)=(x, y)+\frac{1}{2} \mathbf{h}(x, y), \\
& \mathbf{g}_{2}(x, y)=(x, y)-\frac{1}{2} \mathbf{h}(x, y),
\end{aligned}
$$

which gives, again, $\mathbf{g}_{1}(x, y)-\mathbf{g}_{2}(x, y)=\mathbf{h}(x, y)$. 
Having understood these basic principles, let us now illustrate the synthesis of dot trajectories by a few examples.

Example 5. Suppose we wish to synthesize an aperiodic screen superposition whose dot trajectories visually illustrate the vector field $\mathbf{h}(x, y)$ defined by

$$
\mathbf{h}(x, y)=\left(2 x y, y^{2}-x^{2}\right)
$$

[see Fig. 6(a)]. As we have just seen, this can be done, for example, by applying to the layers the following transformations $\mathbf{g}_{1}(x, y)$ and $\mathbf{g}_{2}(x, y)$, which distribute the deformation $\mathbf{h}(x, y)$ in equal parts between the two layers:

$$
\begin{aligned}
\mathbf{g}_{1}(x, y) & =(x, y)+\frac{1}{2} \mathbf{h}(x, y) \\
& =\left(x+x y, y+\frac{1}{2} y^{2}-\frac{1}{2} x^{2}\right), \\
\mathbf{g}_{2}(x, y) & =(x, y)-\frac{1}{2} \mathbf{h}(x, y) \\
& =\left(x-x y, y-\frac{1}{2} y^{2}+\frac{1}{2} x^{2}\right) .
\end{aligned}
$$

And indeed, if we apply the transformations $\mathbf{g}_{1}(x, y)$ and $\mathbf{g}_{2}(x, y)$, respectively, to the two superposed layers, we obtain in the superposition dot trajectories that corre-

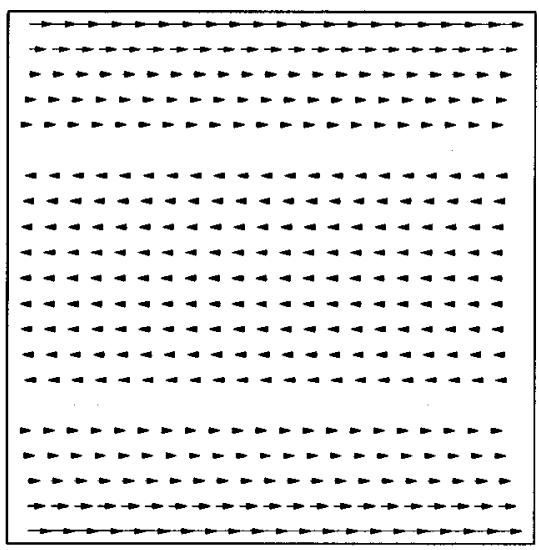

(a)

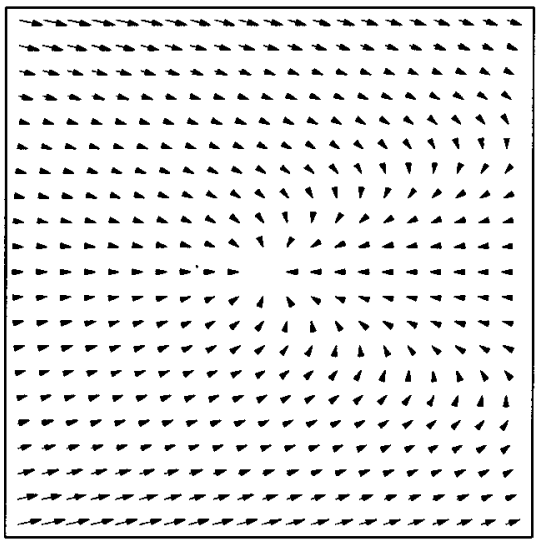

(c) spond to the given vector field $\mathbf{h}(x, y)$. This is clearly illustrated in Fig. 7(a). To improve the visual effect, the transformations $\mathbf{g}_{1}(x, y)$ and $\mathbf{g}_{2}(x, y)$ can be softened by replacing $\mathbf{h}(x, y)$ in both equations by $\varepsilon \mathbf{h}(x, y)$, where $\varepsilon$ is a small positive fraction; this guarantees that the correlation between the two superposed layers remains strong enough to give clearly visible dot trajectories.

Example 6. As a second example, let us choose a system of two differential equations that is well known in physics, such as the nonlinear system describing the behavior of a free undamped pendulum (see, for example, Ref. 19, pp. 181-182 or Ref. 23, pp. 11-17). The system of differential equations is given in this case by

$$
\frac{\mathrm{d}}{\mathrm{d} t} x(t)=y(t), \quad \frac{\mathrm{d}}{\mathrm{d} t} y(t)=-k \sin [x(t)],
$$

where $k$ is a positive constant. The solution curves $[x(t), y(t)]$ of this system are illustrated in Figs. 6(b) and $6(\mathrm{c})$. Since the solution curves of this system are the field lines of the vector field $\mathbf{h}(x, y)=(y,-k \sin x)$ (see Appendix A), we consider the two layer transformations:

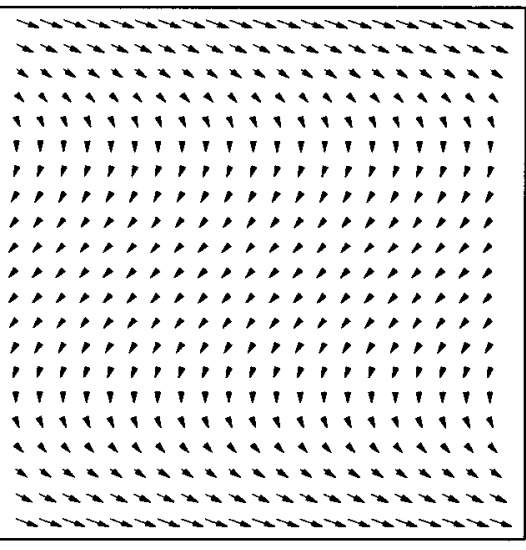

(b)

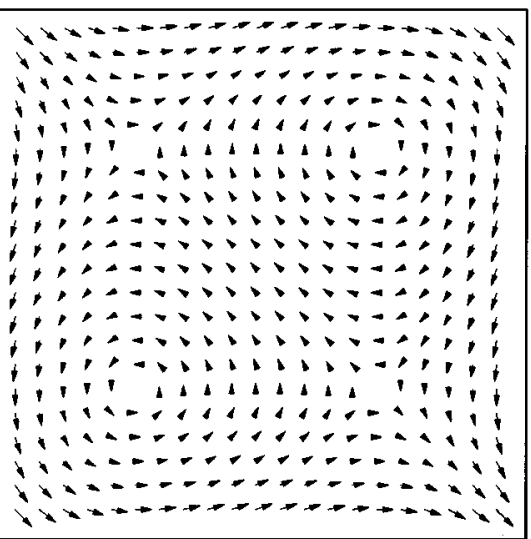

(d)

Fig. 5. Vector field representation of the relative transformation $\mathbf{h}(x, y)=\mathbf{g}_{1}(x, y)-\mathbf{g}_{2}(x, y)$, where (a) $\mathbf{g}_{1}(x, y)$ is a slight parabolic transformation and $\mathbf{g}_{2}(x, y)$ is a slight horizontal shift of $x_{0}$, (b) $\mathbf{g}_{1}(x, y)$ is a slight parabolic transformation and $\mathbf{g}_{2}(x, y)$ is a slight shift of $\left(x_{0}, y_{0}\right)$, (c) $\mathbf{g}_{1}(x, y)$ is a slight parabolic transformation and $\mathbf{g}_{2}(x, y)$ is a slight linear scaling, (d) $\mathbf{g}_{1}(x, y)$ is a slight vertical parabolic transformation with a vertical shift of $y_{0}$ downward, and $\mathbf{g}_{2}(x, y)$ is a slight horizontal parabolic transformation with a horizontal shift of $x_{0}$ to the left Compare with Figs. 3(c), 3(d), 3(e) and 3(f). 




(a)

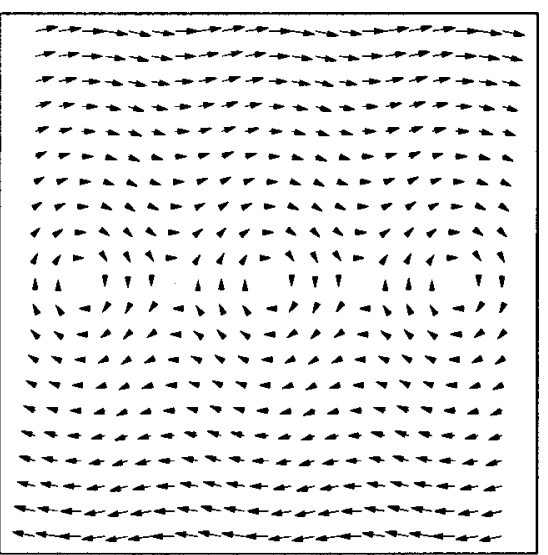

(b)

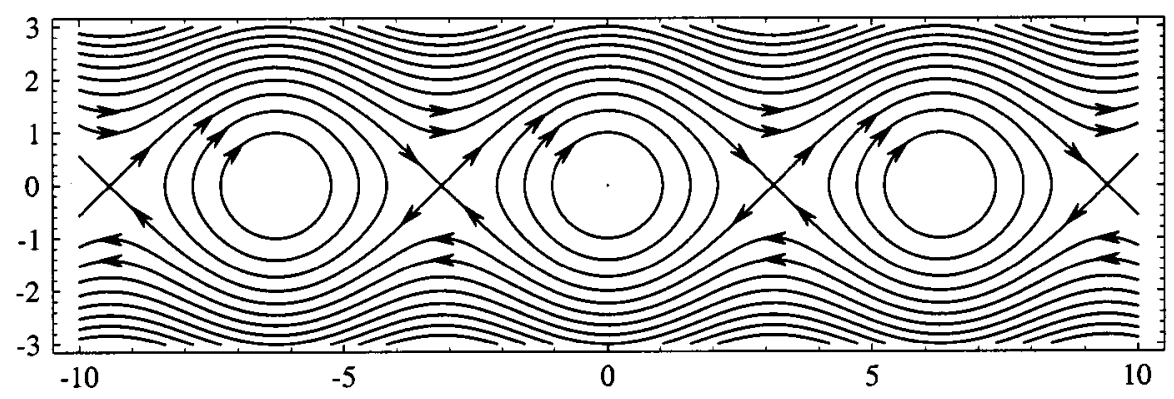

(c)

Fig. 6. (a) Vector field representation of the nonlinear transformation $\mathbf{h}(x, y)=\left(2 x y, y^{2}-x^{2}\right)$. nonlinear system of differential equations describing the behavior of a free undamped pendulum.

(b) Vector field representation of the differential equations of (b), drawn in a larger scale for clarity.

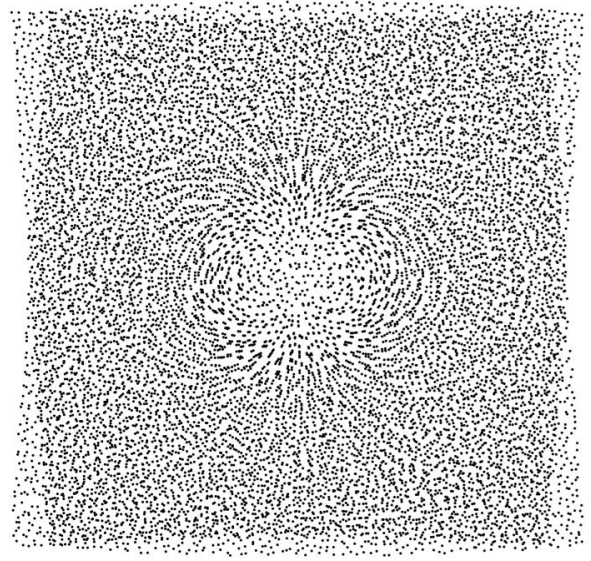

(a)

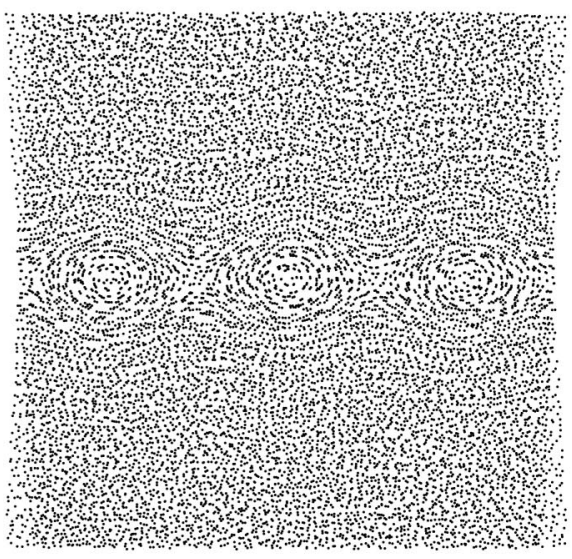

(b)

Fig. 7. Examples of synthesized aperiodic layers that give in their superposition dot trajectories having a given desired shape. (a) Dot trajectories that show the flow lines of the vector field $\mathbf{h}(x, y)=\left(2 x y, y^{2}-x^{2}\right)$, (b) dot trajectories that illustrate the nonlinear system of differential equations describing the behavior of a free undamped pendulum. The transformations applied to the original layers in (a) are $\mathbf{g}_{1}(x, y)=(x, y)+\frac{1}{2} \mathbf{h}(x, y)$ and $\mathbf{g}_{2}(x, y)=(x, y)-\frac{1}{2} \mathbf{h}(x, y)$, and in (b) they are $\mathbf{g}_{1}(x, y)=(x, y)+\frac{1}{2}(y,-k \sin x)$ and $\mathbf{g}_{2}(x, y)=(x, y)-\frac{1}{2}(y,-k \sin x)$. Note the agreement with the vector fields shown in Fig. 6 .

$$
\begin{aligned}
& \mathbf{g}_{1}(x, y)=(x, y)+\frac{1}{2} \mathbf{h}(x, y)=\left(x+\frac{1}{2} y, y-\frac{1}{2} k \sin x\right), \\
& \mathbf{g}_{2}(x, y)=(x, y)-\frac{1}{2} \mathbf{h}(x, y)=\left(x-\frac{1}{2} y, y+\frac{1}{2} k \sin x\right) .
\end{aligned}
$$

And indeed, as shown in Fig. 7(b), if we apply these transformations to our superposed aperiodic screens, we obtain in the superposition dot trajectories that correspond to Fig. 6(b).

These examples illustrate, indeed, how our results can be used to synthesize aperiodic screen superpositions having any desired dot trajectories. It should be repeated, 
however, that the dot trajectories can be visible in the superposition only when the transformations that we apply to the two layers are not too "violent"; otherwise, the correlation between the layers is strongly reduced, and the visual effect is lost.

Finally, it should be noted that this synthesis of dot trajectories is completely independent of the synthesis of Glass patterns having a predetermined intensity profile (gray level distribution), which was described earlier in Ref. 15. That previous contribution explained how to design two random dot screens having an identical dot distribution but different dot shapes (for example, "1"-shaped dots in one layer and tiny pinholes on a black background in the other layer), which generate in their superposition a Glass pattern having a given macroscopic intensity profile (in this example, a Glass pattern having the shape of a considerably magnified " 1 "). In the present paper, on the contrary, we are not interested in the shape of the individual dots in the superposed layers nor in the macroscopic intensity profile of the resulting Glass pattern in the superposition. Rather, we are interested in the geometric transformations that are applied to each of the superposed layers and in their influence on the resulting shapes of the microstructures (dot trajectories) that are generated around the Glass pattern. Obviously, the two approaches can be used independently of each other and can even be combined in order to synthesize in the superposition a Glass pattern having a given intensity profile that is surrounded by any desired dot trajectories.

\section{DOT TRAJECTORIES IN PERIODIC, REPETITIVE, AND APERIODIC CASES}

We now return to the intriguing question that we have already mentioned earlier: Why do we observe dot trajectories only in aperiodic superpositions and not in their corresponding periodic or repetitive superpositions (compare, for example, the left and right cases in each row of Fig. 1)? A careful examination, under a magnifying glass, of the microstructure in a periodic or a repetitive superposition [for example, in Fig. 1(d)] will reveal that similar circular, elliptical, or hyperbolic arrangements of dot pairs do exist about the fixed points in such cases, too. However, in periodic or repetitive cases the dot locations are strongly constrained by an imposed ordering, which limits the degrees of freedom in the microstructure and makes the dot trajectories much less conspicuous than in aperiodic superpositions. Furthermore, this imposed ordering also generates in the superposition a highly visible moiré structure whose presence obscurs the arrangements of the correlated dot pairs in the microstructure. In aperiodic cases the microstructure ordering is not perturbed by any other imposed structures, and therefore the dot trajectories can manifest themselves freely in the superposition.

Note, however, that the dot trajectories are less visible when the correlation between the superposed layers is lower (for example, when a certain amount of random noise is added to the original layers) or when each of the superposed layers consists of elements of a different shape (for example, "1"-shaped elements in one layer and tiny pinholes in the other layer, as in Ref. 15).

\section{CONCLUSIONS}

In the present paper we concentrate on the microstructure dot alignments, known as dot trajectories, that appear in the superpositions of correlated aperiodic dot screens. We show that these dot trajectories visually express the vector field representation of the relative layer transformation $\mathbf{h}(x, y)$ between the two superposed layers, and we show why the explanations based on the absolute layer transformations do not work. Having understood this phenomenon, we then go the other way around and show how one can synthesize aperiodic dot screens that give in their superposition dot trajectories that correspond to any desired vector field $\mathbf{h}(x, y)$ or system of differential equations

$$
\frac{\mathrm{d}}{\mathrm{d} t} x(t)=h_{1}(x(t), y(t)), \quad \frac{\mathrm{d}}{\mathrm{d} t} y(t)=h_{2}(x(t), y(t)) .
$$

Finally, we also explain why dot trajectories are clearly visible in superpositions of aperiodic screens but not in superpositions of periodic screens.

\section{APPENDIX A: MATHEMATICAL INTERPRETATIONS OF A TWO-DIMENSIONAL MAPPING $g(x, y)$}

Consider a system of two equations in two independent variables $x$ and $y$,

$$
u=g_{1}(x, y), \quad v=g_{2}(x, y),
$$

or, in vector notation,

$$
\mathbf{u}=\mathbf{g}(\mathbf{x})
$$

where $\quad \mathbf{x}=(x, y), \quad \mathbf{u}=(u, v), \quad$ and $\quad \mathbf{g}(x, y)$ $=\left[g_{1}(x, y), g_{2}(x, y)\right]$. Clearly, $\mathbf{g}(x, y)$ is a vector function, i.e., a function that returns for each point $(x, y)$ $\in \mathbb{R}^{2}$ a new point $(u, v) \in \mathbb{R}^{2}$.

The mathematical relationship defined by Eqs. (A1) [or alternatively by Eq. (A2)] can be interpreted in several different yet completely equivalent ways, as explained below. Because all of these interpretations are mathematically equivalent, we are free in each case to use any of them, according to our best judgment.

In a first interpretation, we may consider the vector function $\mathbf{g}(x, y)$ of Eq. (A2) a mapping from the $x, y$ plane onto itself.

Alternatively, we may interpret system (A1) or its vector representation (A2) as a change of coordinates in the plane, whereby a new curvilinear coordinate system $u, v$ is defined instead of the original Cartesian coordinate system $x, y$.

Finally, another useful interpretation is obtained by considering the vector function $\mathbf{g}(x, y)$ of (A2) as a vector field. A vector field in $\mathbb{R}^{2}$ is a function $\mathbf{g}(x, y)$ that assigns to each point $(x, y)$ in the $x, y$ plane a vector $(u, v)=\mathbf{g}(x, y)$. Well-known examples in physics are electric or magnetic fields and the gravitation field of the earth, all of which are vector fields (that are defined in the three-dimensional space $\mathbb{R}^{3}$ ).

A vector field in $\mathbb{R}^{2}$ can be illustrated graphically by drawing an arrow emanating from each point $(x, y)$ of the 
$x, y$ plane (or, more practically, from some representative points defined on a given grid within the $x, y$ plane), where the length and the orientation of each arrow indicate the length and the orientation of the vector that has been assigned by $\mathbf{g}(x, y)$ to the point $(x, y)$ [see Fig. 8(a)]. It is important to note, however, that this arrow does not connect the point $(x, y)$ to its destination $\mathbf{g}(x, y)$ under the transformation $\mathbf{g}$ but rather to the point $(x, y)$ $+\mathbf{g}(x, y)$.

To better visualize a vector field, one may draw its trajectories (also known as field lines). Loosely speaking, these are the curves obtained by following the arrows in Fig. 8(a) and joining them into continuous curves in the $x$, $y$ plane [see Fig. 8(b)]. More precisely, trajectories (or field lines) are curves for which the tangent vector to the curve at each point $(x, y)$ is exactly $\mathbf{g}(x, y)$. Thus, at every point $(x, y)$, the direction in which the trajectory runs is determined by the vector $\mathbf{g}(x, y)$. Note that except for points where $\mathbf{g}(x, y)$ is not defined or where $\mathbf{g}(x, y)$ $=\mathbf{0}$, every point in the plane belongs to one and only one trajectory.



(a)
In Cartesian coordinates, the trajectories of a vector field $\mathbf{g}(x, y)$ are given in parametric form by a family of curves $[x(t), y(t)]$ that are solutions of the system of differential equations (see Ref. 24, p. 526),

$$
\frac{\mathrm{d}}{\mathrm{d} t} x(t)=g_{1}(x(t), y(t)), \quad \frac{\mathrm{d}}{\mathrm{d} t} y(t)=g_{2}(x(t), y(t)),
$$

where $t$ is the parameter of each of the curves.

Ways of solving systems of differential equations such as Eqs. (A3) can be found, along with many illustrative examples and figures showing their trajectories, in Chap. 4 of Ref. 19. A complete classification of the different trajectory shapes for linear differential equation systems, including nodes, saddle points, center points, and spirals, can be found in Ref. 19, pp. 176-178; Ref. 25, pp. 551567; and Sec. 1.4 of Ref. 23. A similar classification for nonlinear differential equation systems can be found in Ref. 25, pp. 586-602.



(b)

Fig. 8. (a) Vector field representation of the nonlinear transformation $\mathbf{h}(x, y)=\left(x+a y^{2}, y\right)-(s x, s y)$ with $a=0.001$ and $s$ $=1.0005$. (b) Some field lines (trajectories) of this vector field.

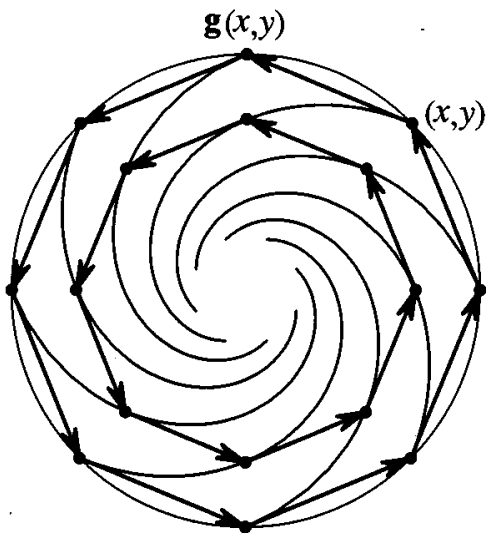

(a)

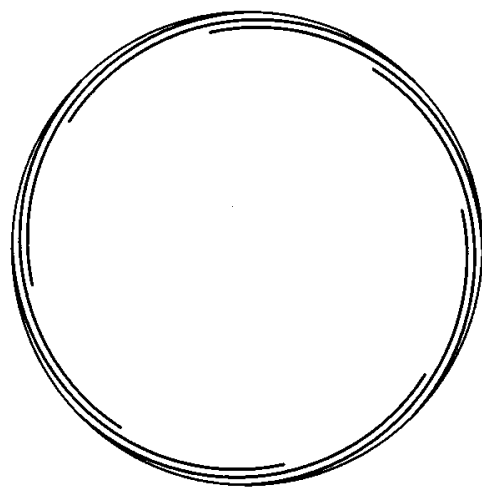

(b)

Fig. 9. (a) The arrow (vector) connecting point $(x, y)$ to its new location $\mathbf{g}(x, y)$ after the application of the rotation transformation $\mathbf{g}$ is not tangential to the circle but rather a chord. Therefore the field line emanating from $(x, y)$ slightly spirals inward, and it is not purely circular. (b) Clearly, the smaller the rotation angle $\alpha$, the closer the field line follows the circle. The rotation angles used in (a) and (b) are, respectively, $45^{\circ}$ and $5^{\circ}$. 


\section{APPENDIX B: DOT TRAJECTORIES AND THE DIFFERENTIAL EQUATIONS}

Suppose that we are given two identical aperiodic dot screens that are superposed precisely on top of each other and that we apply a slight geometric transformation $\mathbf{g}(x, y)$ to one of the two layers and observe the dot trajectories in the resulting superposition. We have seen in Subsection 4.B that the vector field of the relative layer transformation $\mathbf{h}(x, y)=\mathbf{g}(x, y)-(x, y)$ (unlike that of the absolute layer transformation $\mathbf{g}(x, y)$ ] fully corresponds to the dot trajectories that are generated in the screen superposition. The reason is, of course, that the arrows of this vector field connect the departure and the destination points of each screen dot under the transformation $\mathbf{g}(x, y)$.

Now, we remember that the system of differential equations (3) is simply a different representation of the vector field $\mathbf{g}(x, y)$, since its solution curves $[x(t), y(t)]$ express the field lines (trajectories) of the vector field $\mathbf{g}(x, y)$ (see Appendix A). Therefore, by rewriting the system of differential equations (3) for the relative layer transformation $\mathbf{h}(x, y)$ rather than for the absolute layer transformation $\mathbf{g}(x, y)$, we obtain a new system of differential equations,

$$
\frac{\mathrm{d}}{\mathrm{d} t} x(t)=h_{1}(x(t), y(t)), \quad \frac{\mathrm{d}}{\mathrm{d} t} y(t)=h_{2}(x(t), y(t)),
$$

whose solution curves correspond to the field lines of the vector field $\mathbf{h}(x, y)=\mathbf{g}(x, y)-(x, y)$. It can be expected, therefore, that the solution curves of this system of differential equations, unlike those of the original system (3), do correspond to the dot trajectories observed in the superposition. However, it turns out that the resulting solution curves do not always fully agree with the dot trajectories in the superposition. For example, when the layer transformation $\mathbf{g}(x, y)$ is a rotation by angle $\alpha$, the solution curves of the system of differential equations belonging to $\mathbf{h}(x, y)=\mathbf{g}(x, y)-(x, y)$ consist of spirals that converge to the center of rotation (see Ref. 21, p. 144), while the dot trajectories we observe in the screen superposition [Fig. 1(c)] as well as the arrow alignment of the vector field $\mathbf{h}(x, y)$ [Fig. 4(a)] consist of circles surrounding the center of rotation. This discrepancy is explained as follows.

By definition, the field line at each point $(x, y)$ of a vector field is tangent to the arrow emanating from the same point. However, the arrow connecting the point $(x, y)$ to its rotated location $\mathbf{g}(x, y)$ is not tangential to the circle's arc traced by the rotating point; rather, it is a chord connecting the points $(x, y)$ and $\mathbf{g}(x, y)$ on the circumference of the circle. Therefore the field line passing through point $(x, y)$ follows the direction of the chord emanating from $(x, y)$, and hence it is not tangent to the circle but rather oriented slightly inward [see Fig. 9(a)]. This explains why the field lines in this case are, indeed, spirals that converge into the center of rotation, although the arrows of the vector field $\mathbf{h}(x, y)$ are arranged circularly. But such a spiral obviously does not trace out the motion of a screen dot under the rotation transformation $\mathbf{g}(x, y)$, since each dot clearly moves along the circumference of the circle. In other words, although the modified system of differential equations with $\mathbf{h}(x, y)$ rather than $\mathbf{g}(x, y)$ does express the field lines of our vector field $\mathbf{h}(x, y)$, these field lines simply do not represent the circular dot trajectories that we observe in the layer superposition [Fig. 1(c)]. It should be noted, however, that when the rotation angle $\alpha$ is small, the spiral field lines are in fact very close to perfect circles and the discrepancy is negligible [compare the spiral field lines in Figs. 9(a) and 9(b) that correspond, respectively, to $\alpha=45^{\circ}$ and $\alpha=5^{\circ}$ ]. The difference between the spiral field lines and the circular dot trajectories becomes significant only for large angles $\alpha$, but in these cases the visual effect of the dot trajectories is lost anyway, because the correlation between the superposed layers is strongly reduced. On the other hand, the graphic representation of the vector field $\mathbf{h}(x, y)$ in terms of arrows connecting the departure and the destination points of each screen dot under the rotation $\mathbf{g}(x, y)$ [Fig. 4(a)] always remains faithful to the dot trajectories in the superposition (as long as they are visible, as explained in the paragraph following proposition 1 in Subsection 4.B).

Finally, it should be also mentioned that, in general, following the arrows of a vector field by hand in order to get an idea of the shape of the field lines (the solution curves) is not always as easy as it sounds, and it may be sometimes quite misleading. In our present case, for example, one may be tempted to guess from the circular nature of the vector field that by following the arrows, one obtains circular solution curves, although in reality the solution curves are spirals (that just slightly deviate from perfect circles). More details on this point, as well as several illustrated examples, can be found in Ref. 26, pp. $39-42$ and 69.

\section{REFERENCES AND NOTES}

1. Y. Nishijima and G. Oster, "Moiré patterns: their application to refractive index and refractive index gradient measurements,” J. Opt. Soc. Am. 54, 1-5 (1964).

2. D. Tollenaar, "Moiré in halftone printing interference phenomena." The English translation of the original Dutch paper from 1945 is reprinted in Selected Papers on Optical Moiré and Applications, G. Indebetouw and R. Czarnek, eds., Vol. MS64 of SPIE Milestone Series (SPIE Optical Engineering Press, Bellingham, Wash., 1992), pp. 618-633.

3. O. Bryngdahl, "Moiré: formation and interpretation," J. Opt. Soc. Am. 64, 1287-1294 (1974).

4. I. Amidror, The Theory of the Moiré Phenomenon (Kluwer, Dordrecht, The Netherlands, 2000).

5. G. Oster, M. Wasserman, and C. Zwerling, "Theoretical interpretation of moiré patterns," J. Opt. Soc. Am. 54, 169175 (1964).

6. A. W. Lohmann and D. P. Paris, "Variable Fresnel zone pattern," J. Opt. Soc. Am. 6, 1567-1570 (1967).

7. I. Leifer, J. M. Walls, and H. N. Southworth, "The moiré pattern produced by overlapping zone plates," Opt. Acta 20, 33-47 (1973).

8. J. M. Walls and H. N. Southworth, "The moiré pattern formed on superposing a zone plate with a grating or grid," Opt. Acta 22, 591-601 (1975).

9. G. L. Rogers, "A geometrical approach to moiré pattern calculations," Opt. Acta 24, 1-13 (1977).

10. Ref. 4, Chaps. 10 and 11; also published (in part) in I. Amidror, "Fourier-based analysis and synthesis of moirés in the superposition of geometrically transformed periodic structures," J. Opt. Soc. Am. A 15, 1100-1113 (1998). 
11. L. Glass, "Moiré effect from random dots," Nature 223, 578-580 (1969).

12. L. Glass and R. Pérez, "Perception of random dot interference patterns," Nature 246, 360-362 (1973).

13. I. Amidror, "Unified approach for the explanation of stochastic and periodic moirés," J. Electron. Imaging 12, 669681 (2003).

14. I. Amidror, "Glass patterns in the superposition of random line gratings,” J. Opt. A, Pure Appl. Opt. 5, 205-215 (2003).

15. I. Amidror, "Moiré patterns between aperiodic layers: quantitative analysis and synthesis," J. Opt. Soc. Am. A 20, 1900-1119 (2003).

16. The PostScript files have been added at the end of the Moiré Demonstration Kit located at the internet address http://spwww.epfl.ch/books/moire/kit.html.

17. The study of a given phenomenon at different scales is commonplace in physics (and in science in general). More on this subject can be found, for example, in T. Poston and I. Stewart, Catastrophe Theory and Its Applications (Dover, New York, 1978), p. 217, where the different scales of modeling physical phenomena are explained in the context of the flow of fluids.

18. The Fourier analysis of Glass patterns and its global, quali- tative, and quantitative results have been presented separately in Ref. 15 .

19. E. Kreyszig, Advanced Engineering Mathematics, 7th ed. (Wiley, New York, 1993).

20. See Appendix A for more details on the interpretation of such systems of differential equations and on the significance of their solution curves (trajectories). Note that we reserve the term "dot trajectories" for the dot arrangements that occur in random screen superpositions. When confusion may arise we will call the trajectories of differential equations "solution curves."

21. G. Birkhoff and G.-C. Rota, Ordinary Differential Equations, 4th ed. (Wiley, New York, 1989).

22. L. Glass, "Looking at dots," Math. Intell. 24, 37-43 (2002).

23. M. Tabor, Chaos and Integrability in Nonlinear Dynamics: an Introduction (Wiley, New York, 1989).

24. I. N. Bronshtein and K. A. Semendyayev, Handbook of Mathematics (Springer, Berlin, 1997).

25. A. Gray, M. Mezzino, and M. A. Pinsky, Introduction to Ordinary Differential Equations with Mathematica (Springer, New York, 1997).

26. D. Schwalbe and S. Wagon, VisualDSolve: Visualizing Differential Equations with Mathematica (Springer, New York, 1997). 